\title{
ESTIMAÇÃO DO VOLUME DE ÁRVORES DE CLONES DE EUCALIPTO PELO MÉTODO DA SIMILARIDADE DE PERFIS ${ }^{1}$
}

\author{
Márcio Leles Romarco de Oliveira ${ }^{2}$, Helio Garcia Leite ${ }^{3}$, Silvana Lages Ribeiro Garcia ${ }^{4}$, João Carlos Chagas \\ Campos $^{3}$, Carlos Pedro Boechat Soares ${ }^{3}$ e Reynaldo Campos Santana ${ }^{2}$

\begin{abstract}
RESUMO - Os objetivos deste trabalho foram desenvolver e propor um procedimento para quantificação do volume de árvores em plantios clonais de eucalipto não desbastados. Para quantificar o volume de árvores, foram utilizados dados de 2.036 árvores de clones comerciais de eucalipto pertencentes à empresa CAF Santa Bárbara, onde foram ajustadas as equações de volume de cada estrato, sendo este determinado pela região e pelo clone, e as idades dos clones variaram de 4 a 5 anos. Para cada árvore foi ajustado um modelo de afilamento. Posteriormente, foram realizados abates de três árvores de clones novos, que não possuíam equação de volume específica, sendo uma árvore "pequena", uma "média" e uma "grande" em termos de dap e altura total. Com as três árvores cubadas, foi ajustado o modelo de afilamento, considerando-se as três árvores. De posse das estimativas dos parâmetros, foi calculada uma medida de similaridade com os parâmetros das equações de afilamento ajustadas para as 2.036 árvores dos clones comerciais e o parâmetro da equação do clone novo. A árvore do clone comercial que apresentou o menor valor de distância euclidiana, em comparação com o clone novo, foi considerada a mais similar; portanto, a equação de volume desse clone comercial foi usada para estimar o volume de árvores desse novo clone. Tal procedimento foi denominado método da similaridade de perfis. Esse método pode ser usado para estimar volume de árvores dos clones que não têm equações específicas, gerando estimativas semelhantes ao comparar dados observados e estimados, tanto de árvores individuais quanto de todo um talhão.
\end{abstract}

Palavras-chave: Clone de eucalipto, equação de afilamento e equação de volume

\section{ESTIMATE OF THE VOLUME OF EUCALYPT CLONE TREES USING THE PROFILE SIMILARITY METHOD}

\begin{abstract}
The objective of this work was to develop and propose a procedure to quantify the volume of trees in non-thinned Eucalypt clone plantings. To quantify the volume of the trees, data pertaining to 2036 commercial trees of eucalypt clones owned by CAF, Santa Barbara were used, with their volume equations being adjusted for each group, determined by clone and region. For each tree, a taper model was adjusted. For each tree, a taper model was adjusted, followed by the cut of three trees from new clones without specific volume equation, i.e., a "small" tree, an "average" tree, and "a large" tree, in terms of dap and total height. With the three trees cubed, the taper model was adjusted, considering the three trees. Based on the estimates of the parameters, a measure of similarity was calculated for the tape equation parameters adjusted for the 2,036 commercial clone trees and the equation parameter of the new clone. The commercial clone tree presenting the lesser Euclidean distance value when compared with the new clone was considered the most similar; thus, the volume equation of this commercial clone was used estimated the volume of the new clone trees, with this procedure being termed profile similarity method. This method can be used to estimate the tree volume of clones without specific equations, generating similar estimates when comparing observed and estimated, both for individual trees and total stand.
\end{abstract}

Keywords: Eucalypt clone, taper equation, and volume equation.

\footnotetext{
${ }^{1}$ Recebido em 19.09.2007 e aceito para publicação em 26.01.2009.

${ }^{2}$ DepartamentodeEngenhariaFlorestal daUniversidadeFederal dos ValesdoJequitinhonhaeMucuri,UFVJM.E-mail: $<$ marcioromarco@gmail.com>.

${ }^{3}$ Departamento de Engenharia Florestal da UFV. E-mail: <hgleite@ gmail.com>.

${ }^{4}$ Instituto Viçosence de Ensino e Pesquisa, Faculdade de Viçosa - FDV. Viçosa-MG.
} 


\section{INTRODUÇÃO}

A estimativa do volume de árvores individuais é usualmente obtida por meio de equações de volume ou de afilamento. Para gerar essas equações são utilizados dados de cubagem de árvores abatidas ou de árvores cubadas ainda em pé, empregando-se instrumentos específicos como o telerelascópio, pentaprisma, ou um criterion.

A amostragem para geração de equações de volume para florestas equiâneas é feita usualmente por espécie, espaçamento, classe de idade e regime de corte (CAMPOS e LEITE, 2006). Na prática, quanto maior o número de estratos, maior a precisão, porém maior é também o custo de amostragem. No caso de plantios de eucalipto, quando a amostragem é bem planejada é possível complementar a amostra anualmente com o abate de árvores em novas classes de diâmetro. Isso resulta em reduzido número de árvores-amostra ao final da rotação. No entanto, a alternativa de abater árvores em todas as classes de diâmetro, de modo independente, em cada idade, implica grande frequência de árvoresamostra ao final da rotação. Do ponto de vista teórico, isso seria interessante, pois, em alguns casos, o número ideal de árvores-amostra para ajuste de um modelo volumétrico pode chegar a 100 ou 150 árvores (GUIMARÃES et al., 1996). Entretanto, no caso de plantios clonais essa grande frequência pode ser inviável em razão do aumento nos custos pela cubagem de árvores sem necessidade.

Algumas metodologias desenvolvidas podem ser usadas para otimizar o procedimento de cubagem. Leite e Andrade (2004) desenvolveram um método conhecido como método da altura relativa, que pode ser usado para evitar o abate de número excessivo de árvoresamostra. Segundo Gorgens (2006), o uso de redes neurais também pode ser uma técnica para otimizar o processo de cubagem de árvores.

A estratificação para fins de estimação de equações volumétricas é bem estudada quando se trata de plantios de semente. Entretanto, quando se trata de clones, muitas vezes é necessário agrupá-los com base em critérios complementares como classe de solo, classe de precipitação e topografia, entre outras.

Em muitos casos é impossível ou inviável contar com dados de cubagem para geração de equações volumétricas. Quando existe número excessivo de clones plantados, muitos deles com áreas reduzidas, é comum aplicar equações mais genéricas. Uma solução interessante nesses casos é estimar equações específicas para clones mais representativos e utilizar equações genéricas para os demais. Uma equação genérica pode ser aquela ajustada com dados agrupados de vários clones ou uma equação específica ser aplicada a vários outros clones, para os quais não se têm equações. Algumas idéias de agrupamento com base na forma do fuste são encontradas em Campos e Leite (2006).

A estimação de equações volumétricas em empresas do setor privado há muito tempo é prática usual. O principal modelo tem sido o de Schumacher e Hall (1933). Em alguns casos são usadas funções de afilamento, adotando-se modelos polinomiais, como aqueles encontrados em Campos e Leite (2006). Para plantios de semente, esses modelos, especialmente o de volume, têm sido ajustados por espécie, método de regeneração, espaçamento e classe de idade. $\mathrm{O}$ ajuste desse e de outros modelos tem sido feito por procedimentos usuais, como o método de mínimos quadrados ordinários, por procedimentos iterativos, ou utilizando redes neurais. O modelo de Schumacher e Hall tem sido ajustado, na maioria das vezes, por procedimentos de regressão linear.

Com a introdução dos plantios clonais no país a partir de 1979 (FERREIRA, 1992), a quantificação de volume de árvores passou a ser, de certa forma, um novo desafio. Esse desafio existe devido à dinâmica que ocorre envolvendo a introdução de clones anualmente.

A maioria das empresas florestais tem número variado de materiais genéticos (clones) plantados. Às vezes, é plantado um material genético, e antes da colheita sua substituição já está definida para o próximo ciclo. Independentemente dessa dinâmica, é necessário que se tenham todas as estimativas de volume de todos os materiais genéticos.

O objetivo deste trabalho consistiu em desenvolver e propor um procedimento para quantificação do volume de árvores em plantios clonais de eucalipto.

\section{MATERIAL E MÉTODOS}

\subsection{Descrição dos dados}

Os dados utilizados neste trabalho foram obtidos de povoamentos de Eucalyptus sp., pertencentes à empresa CAF-Santa Bárbara Ltda., que faz parte do grupo ARCELOR.Aempresa tem como atividade comercial 
a produção de carvão vegetal. Os plantios estão presentes em Minas Gerais e Bahia, sendo distribuídos nas seguintes regiões: Teixeira de Freitas (Região da Bahia - RBA); Martinho Campos (Região Centro-Oeste-RCO); Carbonita (Região Norte - RNO); e Coronel Fabriciano e Ponte Alta (Região do Rio Doce - RRD).

Foram utilizados dados de 2036 árvores abatidas e cubadas no período de abril de 2002 a maio de 2006, com idades variando de 4 a 5 anos. Os diâmetros ao longo do fuste foram medidos nas posições de 0,10 $\mathrm{m}$ e $1,30 \mathrm{~m}$, e a partir desse ponto as seções foram medidas de 1,0 em 1,0 m até a altura onde se encontrava o diâmetro com casca de cerca de $2,5 \mathrm{~cm}$. Os estratos para fins de cubagem foram definidos pela região e material genético (clone), totalizando 45 estratos (Tabela 1). Foram cubadas, em média, cinco árvores por classe de dap (diâmetro a 1,30 m de altura) com amplitude de 2,0 cm, sendo observada uma variação de dap de 3,9 a $26,1 \mathrm{~cm}$ e, em altura, de 5,6 a 33,8 m.

Tabela 1 - Número de árvores-amostra $(N)$ e amplitude dos diâmetros (dap) e das alturas totais (Ht) das árvores cubadas por estrato

Table 1 - Number of sample-trees (n), amplitude of the diameter (dap) and total heights (Ht) of the trees cubed by group

\begin{tabular}{|c|c|c|c|c|c|c|c|}
\hline \multirow[t]{2}{*}{ Estrato } & \multirow[b]{2}{*}{$N$} & \multicolumn{3}{|c|}{ dap $(\mathrm{cm})$} & \multicolumn{3}{|c|}{$H t(\mathrm{~m})$} \\
\hline & & Mínimo & Médio & Máximo & Mínima & Média & Máxima \\
\hline RBA CAF05 & 51 & 6,4 & 18,0 & 26,1 & 10,7 & 24,2 & 32,0 \\
\hline RBA CAF08 & 45 & 4,8 & 14,7 & 22,0 & 7,8 & 21,1 & 24,9 \\
\hline RBA CAF09 & 50 & 6,7 & 17,8 & 26,1 & 13,0 & 24,8 & 32,8 \\
\hline RBA CAF11 & 70 & 5,1 & 16,4 & 26,1 & 9,7 & 21,7 & 31,2 \\
\hline RBA CAF21 & 68 & 4,8 & 17,5 & 23,9 & 7,2 & 21,9 & 28,6 \\
\hline RBA CAF25 & 42 & 4,5 & 8,2 & 11,1 & 6,7 & 11,4 & 15,4 \\
\hline RBA CAF26 & 42 & 5,1 & 14,4 & 20,1 & 8,7 & 19,8 & 24,7 \\
\hline RBA CAF 27 & 52 & 5,7 & 16,9 & 23,9 & 10,6 & 22,5 & 29,7 \\
\hline RBA CAF28 & 40 & 4,8 & 15,0 & 20,7 & 9,1 & 20,7 & 26,6 \\
\hline RBA CAF 29 & 55 & 4,8 & 17,3 & 25,1 & 8,7 & 24,1 & 33,8 \\
\hline RBA CAF30 & 50 & 6,4 & 17,9 & 26,1 & 12,1 & 24,1 & 30,0 \\
\hline RBA CAF31 & 29 & 4,5 & 11,1 & 15,9 & 6,8 & 14,2 & 15,9 \\
\hline RBA CAF36 & 124 & 4,8 & 14,2 & 22,0 & 8,9 & 21,8 & 28,0 \\
\hline RBA CAF37 & 37 & 5,4 & 13,5 & 18,1 & 6,7 & 19,1 & 24,8 \\
\hline RBA CAF37 C.ERVA & 39 & 5,4 & 15,1 & 19,7 & 6,0 & 22,8 & 27,6 \\
\hline RBACAF37_PALHAL & 51 & 5,7 & 15,8 & 22,0 & 7,7 & 22,6 & 31,4 \\
\hline RBA CAF44 & 91 & 4,5 & 13,5 & 18,1 & 8,8 & 19,4 & 25,3 \\
\hline RBA CAF46 & 133 & 5,1 & 16,1 & 22,0 & 7,5 & 19,1 & 25,3 \\
\hline RCO CAF03 & 19 & 5,4 & 13,9 & 20,2 & 6,6 & 16,0 & 24,5 \\
\hline RCO CAF 15 & 40 & 5,4 & 11,9 & 16,9 & 7,4 & 15,0 & 23,1 \\
\hline RCO CAF19 & 35 & 5,4 & 13,7 & 19,7 & 6,5 & 17,9 & 24,5 \\
\hline RCO CAF21 & 12 & 5,7 & 12,6 & 19,7 & 7,9 & 16,0 & 19,6 \\
\hline RCO CAF26 & 34 & 4,6 & 11,1 & 15,9 & 8,7 & 16,2 & 23,0 \\
\hline RCO CAF28 & 34 & 4,6 & 10,1 & 14,3 & 9,2 & 14,9 & 22,0 \\
\hline RCO CAF37 & 34 & 5,1 & 11,5 & 17,5 & 7,2 & 14,1 & 23,8 \\
\hline RCO CAF46 & 51 & 5,7 & 15,8 & 21,0 & 7,3 & 18,2 & 28,0 \\
\hline RNO CAF22 & 36 & 5,6 & 11,1 & 15,4 & 9,0 & 15,1 & 21,5 \\
\hline RNO CAF42 & 39 & 4,8 & 11,1 & 15,6 & 6,8 & 14,8 & 22,5 \\
\hline RNO CAF47 & 36 & 4,8 & 11,2 & 15,3 & 6,9 & 16,7 & 23,1 \\
\hline RNO CAF49 & 46 & 4,7 & 10,8 & 16,0 & 7,7 & 16,3 & 24,3 \\
\hline RNO CAF50 & 40 & 5,0 & 10,9 & 15,3 & 8,8 & 17,4 & 23,3 \\
\hline RRD CAF01 & 53 & 3,9 & 11,1 & 17,3 & 7,7 & 15,3 & 20,8 \\
\hline RRD CAF11 & 39 & 4,1 & 10,5 & 15,5 & 7,4 & 14,0 & 18,8 \\
\hline RRD CAF13 & 44 & 4,5 & 12,0 & 17,5 & 7,7 & 15,7 & 21,1 \\
\hline RRD CAF16 & 25 & 5,0 & 9,6 & 13,5 & 7,9 & 13,3 & 17,5 \\
\hline RRD CAF25 & 47 & 4,3 & 10,4 & 15,2 & 8,5 & 15,1 & 20,1 \\
\hline RRD CAF 26 & 20 & 5,7 & 10,2 & 13,7 & 11,1 & 14,8 & 17,5 \\
\hline RRD CAF 28 & 51 & 4,5 & 10,8 & 16,0 & 7,4 & 14,3 & 18,8 \\
\hline RRD CAF31 & 20 & 6,0 & 10,1 & 12,7 & 10,8 & 13,8 & 17,2 \\
\hline RRD CAF36 & 34 & 4,8 & 12,0 & 17,8 & 9,0 & 17,4 & 22,8 \\
\hline RRD CAF37 & 40 & 5,1 & 10,0 & 12,4 & 8,3 & 14,6 & 17,4 \\
\hline RRD CAF43 & 23 & 4,1 & 8,5 & 12,9 & 5,6 & 10,4 & 16,1 \\
\hline RRD CAF46 & 46 & 5,4 & 10,8 & 14,3 & 10,2 & 15,1 & 19,0 \\
\hline RRD CAF47 & 25 & 4,2 & 9,6 & 13,4 & 8,4 & 13,9 & 18,1 \\
\hline RRD CAF51 & 20 & 5,1 & 9,8 & 13,1 & 9,9 & 14,3 & 16,5 \\
\hline
\end{tabular}




\subsection{Equações de volume}

O volume total observado com casca de cada árvoreamostra foi obtido pelo método de Smalian (HUSCH et al., 2003). De posse dos dados de volume, o modelo de Schumacher e Hall (1933) foi ajustado para cada estrato, na forma:

$$
\operatorname{Ln} V=\beta_{0}+\beta_{1} \operatorname{Lndap}+\beta_{2} \operatorname{Ln} H t+\varepsilon
$$

em que $\mathrm{Ln}=$ logaritmo neperiano; $V=$ volume comercial com casca, em m³ $\beta_{i}=$ parâmetros do modelo, $\mathrm{i}=0,1$, e 2; dap = diâmetro a $1,30 \mathrm{~m}$ de altura solo, em cm; $H t=$ altura total, em $\mathrm{m} ; \varepsilon=$ erro aleatório, sendo $\varepsilon$ $\sim \mathrm{N}\left(0, \sigma^{2}\right)$.

Esses ajustes e essas equações foram fornecidos pela empresa. Conforme sugerido por Campos et al. (1985), foi avaliada em análises prévias e não confirmada a ocorrência de discrepância logarítmica.

\subsection{Procedimento proposto}

Com os dados das árvores cubadas foi ajustado o modelo de afilamento desenvolvido por Kozak et al. (1969) para cada árvore, conforme relação funcional a seguir:

$$
\left(\frac{d_{i}}{d a p}\right)^{2}=\beta_{0}+\beta_{1} \frac{h_{i}}{H t}+\beta_{2}\left(\frac{h_{i}}{H t}\right)^{2}+\varepsilon
$$

em que $d_{i}=$ diâmetro na altura $h_{i}$, em cm; dap = diâmetro a $1,30 \mathrm{~m}$ de altura, em $\mathrm{cm} ; h_{i}=$ altura em que ocorre o diâmetro $d_{i}$, em m; $H t=$ altura total, em m; $\beta_{i}=$ parâmetros da regressão (i=0, 1, e 2); e $\varepsilon=$ erro aleatório, sendo $\varepsilon \sim \mathrm{N}\left(0, \sigma^{2}\right)$.

O modelo de Kozak et al. (1969) foi utilizado por causa da sua facilidade de ajuste e emprego (CAMPOS e LEITE, 2006). Outros modelos (DEMAERSCHALK, 1972; BURKHART, 1977; BIGING, 1984; GARAY, 1979) foram testados, porém, para algumas árvores eles não resultaram em ajustes adequados do ponto de vista estatístico, redundando em estimativas e parâmetros inconsistentes.

Para outros oito clones novos, o modelo foi ajustado utilizando-se dados de três árvores (uma "pequena", uma "média" e outra "grande"), representando locais com qualidades inferior, média e superior. Conforme citado anteriormente, esses clones não tinham dados suficientes para representar um estrato de cubagem, como os apresentados na Tabela 1 .

R. Árvore, Viçosa-MG, v.33, n.1, p.133-141, 2009
Com os parâmetros estimados do modelo de Kozak para todas as árvores, foi empregado um procedimento para calcular a similaridade entre os parâmetros dos $n$ clones e $n$ árvores. Para isso foi utilizada a distância euclidiana (CRUZ et al., 2004):

$$
d_{i i^{\prime}}=\sqrt{\sum_{j=1}^{p}\left(X_{i j}-X_{i^{\prime} j^{\prime}}\right)^{2}}
$$

em que $d_{i j}=$ distância euclidiana entre dois clones $i$ e i'; e X $\mathrm{ij}_{\mathrm{ij}} \mathrm{X}_{\mathrm{i} \mathrm{i}^{\mathrm{j}}}=$ observação no i-ésimo clone $(\mathrm{i}=1,2, \ldots, n)$ em referência ao j-ésimo parâmetro $(\mathrm{j}=1,2, \ldots, p)$.

De posse da matriz da distância euclidiana determinou-se, pelo menor valor da distância, à qual árvore o clone novo era mais similar, em comparação com as árvores dos clones comerciais.

Após a determinação da árvore com maior similaridade, foi verificado à qual estrato (clone e região) o novo clone pertencia, bem como estimou-se o volume utilizando a equação de Schumacher e Hall do respectivo estrato. A estimativa do volume foi, então, feita das três árvores empregadas no ajuste do modelo de afilamento.

O procedimento descrito e proposto neste estudo foi denominado "Método da Similaridade de Perfis".

\subsection{Verificação do Método da Similaridade de Perfis}

O teste F (GRAYBILL, 1976), em nível de 5\% de probabilidade, foi utilizado para comparar as estimativas dos volumes obtidas através da equação de Schumacher e Hall, com os correspondentes volumes observados de cada árvore dos clones novos.

Posteriormente foram sorteadas três árvores, com dap relativamente pequeno, médio e grande, de dois clones comerciais. As outras árvores desses clones foram excluídas para a análise. O método da similaridade de perfis foi aplicado a cada clone sorteado. Em seguida foi empregada a equação de Schumacher e Hall do estrato de cubagem, determinado através da menor distância euclidiana. Foram geradas as estimativas do volume das três árvores escolhidas, além das outras árvores do clone sorteado. Os volumes estimados das árvores com os seus volumes observados foram comparados pelo teste F (GRAYBILL, 1976) em nível de 5\% de probabilidade. Essa comparação foi dos dois clones separadamente. 


\subsection{Validação do Método da Similaridade de Perfis}

A validação foi realizada com dados independentes para avaliar a aplicabilidade do método da similaridade de perfis. Foram utilizados dados de Eucalyptus sp. coletados em uma empresa localizada na região norte de Minas Gerais, distribuídos em 20 estratos, representados por 14 clones e três projetos. Dessa forma, três árvores foram selecionadas ao acaso, sendo uma árvore grande, uma média e uma pequena, em termos de altura e diâmetro. Abatidas essas árvores, foi realizado todo o procedimento de cubagem já descrito. Posteriormente, com a equação de Schumacher e Hall (1933) indicada para usar no estrato (Clone-Projeto) considerado novo, foram comparados os volumes utilizando-se a equação específica do clone e a equação proposta. Essa comparação se deu em nível de talhão. Nesse caso, optou-se por usar o teste Qui-quadrado (NESBITT, 1995), pelo fato de os dados não apresentarem normalidade.

\section{RESULTADOS E DISCUSSÃO}

\subsection{Análise do Método da Similaridade de Perfis}

As equações de volume obtidas dos clones comerciais fornecidas pela empresa foram analisadas e apresentaram estimativas precisas e livres de tendência. Isso foi comprovado por meio de análises de resíduos, informações essas disponibilizadas pela empresa.

Utilizando o método da similaridade de perfis com a estimativa dos parâmetros do modelo de Kozak e a distância euclidiana, verificou-se que as estimativas dos parâmetros do modelo de afilamento dos clones novos com os clones comerciais foram bem próximas, apresentando variação percentual, para mais ou para menos, menor que 2,0\% para todos os parâmetros, à exceção do clone CAF18 na região RCO, que apresentou variação percentual maior que 6,0\% (Tabela 2).

Tabela 2 - Estimativas dos parâmetros $\left(\beta_{0}, \beta_{1}\right.$ e $\left.\beta_{2}\right)$ do modelo de Kozak e distância euclidiana, em que controle $1=$ clone novo, 2 = clone comercial e IGUA = igualdade porcentual entre o parâmetro do clone novo e o do clone comercial

Table 2 - Estimates of the parameters $\left(\beta_{0}, \beta_{1}\right.$ and $\left.\beta_{2}\right)$ of the Kozak model and Euclidean distance where control $1=$ new clone, $2=$ commercial clone and IGUA = percent equality between the parameter of the new clone and that of the commercial clone

\begin{tabular}{|c|c|c|c|c|c|c|}
\hline Controle & Região & Material Genético & $\hat{\beta}_{0}$ & $\hat{\beta}_{1}$ & $\hat{\beta}_{2}$ & Distância Euclidiana \\
\hline 1 & $\mathrm{RCO}$ & CAF07 & 1,431462 & $-2,988481$ & 1,696123 & 0,0442 \\
\hline \multirow[t]{2}{*}{2} & RBA & CAF37 & 1,414698 & $-2,948366$ & 1,688150 & \\
\hline & & IGUA\% & 98,83 & 98,66 & 99,53 & \\
\hline 1 & $\mathrm{RCO}$ & CAF14 & 1,397291 & $-3,068700$ & 1,834764 & 0,0147 \\
\hline \multirow[t]{2}{*}{2} & RRD & CAF47 & 1,384251 & $-3,063899$ & 1,830050 & \\
\hline & & IGUA \% & 99,07 & 99,84 & 99,74 & \\
\hline 1 & $\mathrm{RCO}$ & CAF17 & 1,376763 & $-2,689106$ & 1,429119 & 0,0153 \\
\hline \multirow[t]{2}{*}{2} & RNO & CAF22 & 1,365662 & $-2,698904$ & 1,425282 & \\
\hline & & IGUA \% & 99,19 & 100,36 & 99,73 & \\
\hline 1 & $\mathrm{RCO}$ & CAF18 & 1,424884 & $-3,104030$ & 1,880620 & 0,0417 \\
\hline \multirow[t]{2}{*}{2} & $\mathrm{RCO}$ & CAF03 & 1,512080 & $-3,538107$ & 2,145660 & \\
\hline & & IGUA \% & 106,12 & 113,98 & 114,09 & \\
\hline 1 & $\mathrm{RCO}$ & CAF35 & 1,244618 & $-2,146092$ & 0,939003 & 0,0121 \\
\hline \multirow[t]{2}{*}{2} & $\mathrm{RCO}$ & CAF15 & 1,256092 & $-2,142208$ & 0,938540 & \\
\hline & & IGUA $\%$ & 100,92 & 99,82 & 99,95 & \\
\hline 1 & $\mathrm{RCO}$ & CAF38 & 1,282639 & $-2,601971$ & 1,468947 & 0,0328 \\
\hline \multirow[t]{2}{*}{2} & RBA & CAF37_PALHAL & 1,266544 & $-2,589846$ & 1,494779 & \\
\hline & & IGUA \% & 98,75 & 99,53 & 101,76 & \\
\hline 1 & $\mathrm{RCO}$ & CAF45 & 1,399189 & $-2,880068$ & 1,631849 & 0,0228 \\
\hline \multirow[t]{2}{*}{2} & RNO & CAF50 & 1,417666 & $-2,870354$ & 1,641103 & \\
\hline & & IGUA \% & 101,32 & 99,66 & 100,57 & \\
\hline 1 & RRD & CAF409 & 1,426634 & $-2,934853$ & 1,709917 & 0,0160 \\
\hline \multirow[t]{2}{*}{2} & RRD & CAF46 & 1,428854 & $-2,935601$ & 1,725758 & \\
\hline & & IGUA \% & 100,16 & 100,03 & 100,93 & \\
\hline
\end{tabular}

R. Árvore, Viçosa-MG, v.33, n.1, p.133-141, 2009 
A Figura 1 foi elaborada utilizando-se dados de diâmetro e altura da maior árvore do clone novo como referência e estimando os valores de $d$. dap $^{-1}$ com a equação ajustada pelo modelo de Kozak, da árvore do clone comercial determinada como semelhante. Os pontos no gráfico representam o clone novo e a linha, a estimativa obtida pela equação do clone comercial semelhante. Todos os clones tinham formas distintas de fuste, e as árvores dos clones comerciais que foram determinadas como semelhantes permitiram representar, com eficiência, as respectivas formas. Como determinado na Tabela 2, o clone CAF18 que teve como clone comercial semelhante o CAF03, ambos na região RCO, apresentou desempenho inferior; no caso, houve diferença na parte superior do fuste, que representa menor volume comercial.

\subsection{Verificação do Método da Similaridade de Perfis}

Após a determinação do estrato de cubagem ao qual o novo clone pertencia, foi calculado o volume total desse estrato utilizando-se a equação de Schumacher e Hall e comparado com o seu volume observado. Isso foi feito em todos os clones novos e por árvore (Tabela 3 ).

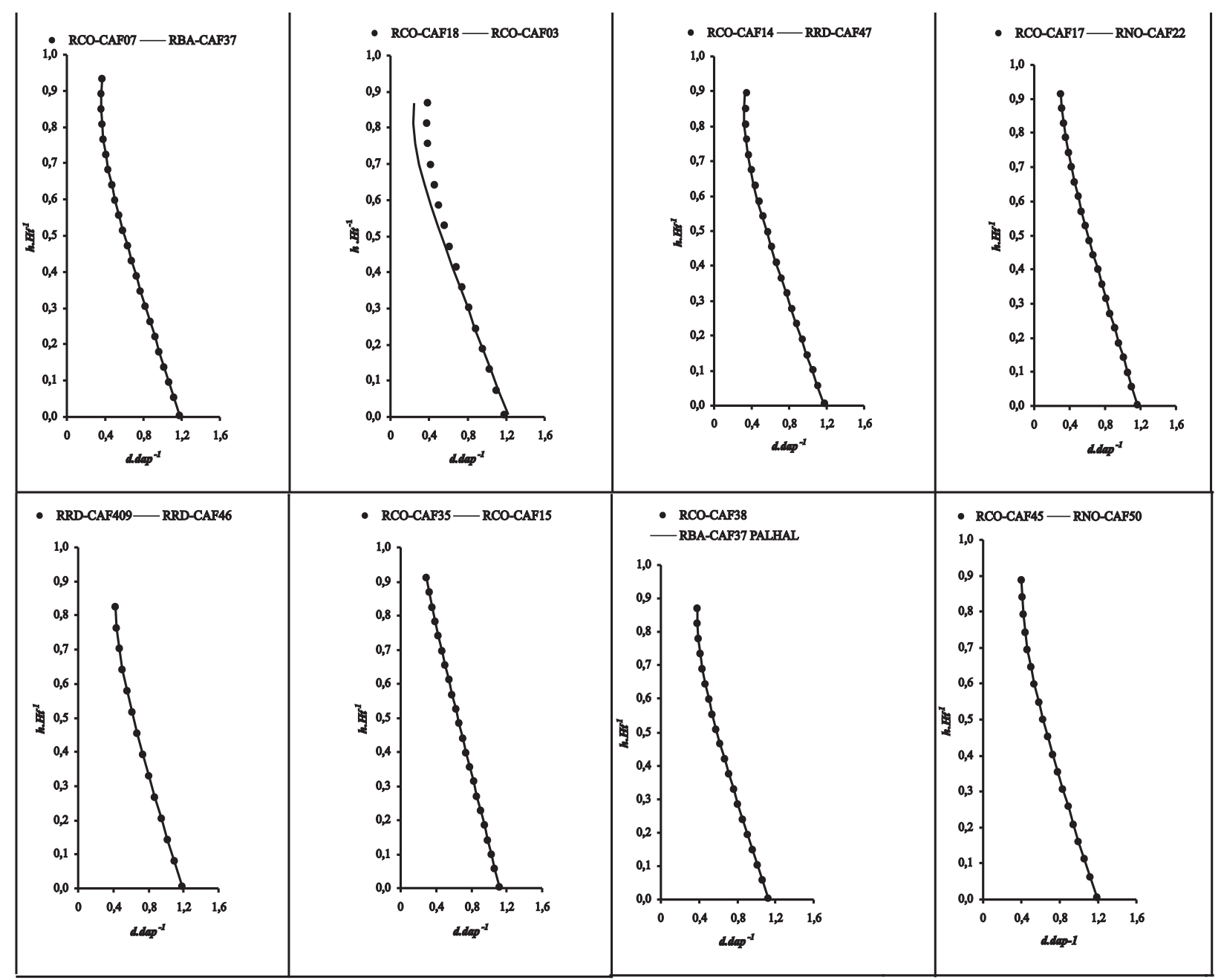

Figura 1 - Análise do perfil de árvores dos clones, obtidos pela equação de afilamento, em que os pontos representam estimativa da maior árvore do clone novo; e a linha representa a estimativa, utilizando-se a equação da árvore do clone comercial semelhante.

Figure 1 -Analysis of the profile of the clone trees, obtained through the taper equation, where the points represent the estimates of the new clone's largest tree; and the line represents the estimate using the similar commercial clone's tree equation.

R. Árvore, Viçosa-MG, v.33, n.1, p.133-141, 2009 
Tabela 3 - Estimativa de dap $(\mathrm{cm}), H t(\mathrm{~m})$, volume observado $\left(\mathrm{m}^{3}\right)$, volume estimado pela equação de Schumacher e Hall do clone semelhante e resíduo $100(1)$, em \% , por material genético e por região

Table 3 - Estimate of dap $(\mathrm{cm}), H t(\mathrm{~m})$, volume observed $\left(\mathrm{m}^{3}\right)$, volume estimated by the Schumacher and Hall equation of the similar clone and residue, in \%, per genetic material and by region

\begin{tabular}{|c|c|c|c|c|c|c|}
\hline Região & $\begin{array}{l}\text { Material } \\
\text { Genético }\end{array}$ & $d a p(\mathrm{~cm})$ & $H t(\mathrm{~m})$ & $\begin{array}{c}\text { Volume Observado } \\
\left(\mathrm{m}^{3}\right)\end{array}$ & $\begin{array}{c}\text { Volume Estimado } \\
\left(\mathrm{m}^{3}\right)\end{array}$ & Resíduo \\
\hline $\mathrm{RCO}$ & CAF38 & 14,32 & 18,90 & 0,1333 & 0,1407 & 5,55 \\
\hline $\mathrm{RCO}$ & CAF38 & 13,05 & 16,30 & 0,0943 & 0,1001 & 6,15 \\
\hline $\mathrm{RCO}$ & CAF38 & 19,10 & 22,20 & 0,2816 & 0,2899 & 2,95 \\
\hline $\mathrm{RCO}$ & CAF14 & 5,41 & 6,50 & 0,0072 & 0,0076 & 5,56 \\
\hline $\mathrm{RCO}$ & CAF14 & 14,01 & 17,30 & 0,1236 & 0,1154 & $-6,63$ \\
\hline $\mathrm{RCO}$ & CAF14 & 21,01 & 22,70 & 0,3081 & 0,3228 & 4,77 \\
\hline RRD & CAF409 & 5,54 & 8,20 & 0,0099 & 0,0084 & $-15,15$ \\
\hline RRD & CAF409 & 8,37 & 15,80 & 0,0448 & 0,0390 & $-12,95$ \\
\hline RRD & CAF409 & 11,40 & 16,10 & 0,0707 & 0,0724 & 2,40 \\
\hline RCO & CAF17 & 5,41 & 9,80 & 0,0103 & 0,0116 & 12,62 \\
\hline $\mathrm{RCO}$ & CAF17 & 16,87 & 21,20 & 0,2430 & 0,2164 & $-10,95$ \\
\hline $\mathrm{RCO}$ & CAF17 & 19,10 & 23,30 & 0,2910 & 0,3011 & 3,47 \\
\hline $\mathrm{RCO}$ & CAF07 & 5,73 & 9,70 & 0,0103 & 0,0111 & 7,77 \\
\hline $\mathrm{RCO}$ & CAF07 & 10,19 & 14,30 & 0,0553 & 0,0526 & $-4,88$ \\
\hline $\mathrm{RCO}$ & CAF07 & 18,78 & 23,90 & 0,2953 & 0,3105 & 5,15 \\
\hline $\mathrm{RCO}$ & CAF45 & 4,77 & 8,10 & 0,0060 & 0,0071 & 18,33 \\
\hline $\mathrm{RCO}$ & CAF45 & 7,96 & 8,60 & 0,0202 & 0,0190 & $-5,94$ \\
\hline $\mathrm{RCO}$ & CAF45 & 17,51 & 20,60 & 0,2106 & 0,2096 & $-0,47$ \\
\hline $\mathrm{RCO}$ & CAF18 & 13,05 & 15,60 & 0,0907 & 0,0929 & 2,43 \\
\hline $\mathrm{RCO}$ & CAF18 & 6,68 & 11,20 & 0,0178 & 0,0198 & 11,24 \\
\hline RCO & CAF18 & 18,40 & 17,60 & 0,2030 & 0,1939 & $-4,48$ \\
\hline $\mathrm{RCO}$ & CAF35 & 7,32 & 11,30 & 0,0223 & 0,0219 & $-1,79$ \\
\hline $\mathrm{RCO}$ & CAF35 & 16,55 & 19,80 & 0,2133 & 0,1959 & $-8,16$ \\
\hline $\mathrm{RCO}$ & CAF35 & 21,01 & 23,40 & 0,3417 & 0,3729 & 9,13 \\
\hline
\end{tabular}

Pelo teste $\mathrm{F}$ ( $\mathrm{F}$ calculado $=1,38$ a $5 \%$ de probabilidade), foi verificado que os volumes estimados foram semelhantes aos observados, comprovando a eficiência do método da similaridade de perfis.

A Tabela 4 contém dados de dois clones comerciais sorteados e a região a que eles pertencem. Após o sorteio, foram separadas três árvores de cada clone, seguindo-se o porte, isto é, uma árvore de portes baixo, médio e grande.

Com esses dados foi estimada a equação de Kozak para cada estrato e, posteriormente, plicado o método da similaridade de perfis para determinar qual árvore, entre aquelas dos clones comerciais restantes, era a mais semelhante. Diante disso, determinou-se a qual novo estrato esses clones pertenciam (Tabela 5).

$\mathrm{Na}$ Figura 2 foram plotados os volumes observados de cada árvore dos estratos sorteados, bem como realizadas as estimativas dos volumes individuais, utilizando-se a equação de Schumacher e Hall do estrato semelhante.
Tabela 4 - Valores de dap $(\mathrm{cm})$ e altura total $(\mathrm{m})$ por árvore e por estratos dos clones sorteados

Table 4-Values of dap (cm) and total height $(\mathrm{m})$ per tree and drafted clone groups

\begin{tabular}{llccc}
\hline Região & $\begin{array}{l}\text { Material } \\
\text { Genético }\end{array}$ & Árvore & dap $(\mathrm{cm})$ & $H t(\mathrm{~m})$ \\
\hline \multirow{2}{*}{ RRD } & & 1 & 6,6 & 8,7 \\
& CAF28 & 2 & 11,1 & 13,8 \\
& & 3 & 15,8 & 17,9 \\
\multirow{2}{*}{ RCO } & CAF15 & 2 & 5,7 & 8,2 \\
& & 3 & 10,2 & 10,8 \\
\hline
\end{tabular}

A equação do estrato semelhante aplicado no estrato RRD-CAF28 teve tendência de superestimar o volume, porém essa tendência não influenciou o teste $\mathrm{F}$ (F calculado $=1,31)$, que resultou em não-significativo a $5 \%$ de probabilidade (Figura 2A). No clone RCOCAF15 (Figura 2B), não houve qualquer tendência, sendo o teste $\mathrm{F}$ ( $\mathrm{F}$ calculado $=1,23$ ) também nãosignificativo, ou seja, nos dois estratos avaliados o volume observado foi igual ao volume estimado pela equação de volume do estrato semelhante.

R. Árvore, Viçosa-MG, v.33, n.1, p.133-141, 2009 
Tabela 5 - Estimativas dos parâmetros $\left(\beta_{0}, \beta_{1}\right.$ e $\left.\beta_{2}\right)$ do modelo de Kozak e distância euclidiana, em que controle $1=$ clone comercial considerado novo, $2=$ clone comercial e IGUA = igualdade porcentual entre o parâmetro do clone comercial considerado novo e o do clone comercial

Table 5 - Estimates of the parameters $\left(\beta_{0}, \beta_{1}\right.$ e $\left.\beta_{2}\right)$ of the Kozak model and Euclidean distance where: control $1=$ commercial clone considered new, 2 = commercial clone and IGUA = percent equality between the parameter of the commercial clone considered new and that of the commercial clone

\begin{tabular}{ccccccc}
\hline Controle & Região & Material Genético & $\hat{\beta}_{0}$ & $\hat{\beta}_{1}$ & $\hat{\beta}_{2}$ & Distância Euclidiana \\
\hline 1 & RRD & CAF28 & 1,3690 & $-2,9178$ & 1,7610 & 0,0375 \\
2 & RRD & CAF47 & 1,3709 & $-2,9109$ & 1,7242 & \\
& & IGUA\% & 100,14 & 99,76 & 97,91 & 0,0529 \\
1 & RCO & CAF15 & 1,4077 & $-2,7567$ & 1,4586 & 1,5098 \\
2 & RNO & CAF22 & 1,3973 & $-2,7648$ & 103,51 & \\
& & IGUA \% & 99,26 & 100,29 & & \\
\hline
\end{tabular}

\subsection{Validação do Método da Similaridade de Perfis}

Na Tabela 6, apresenta-se o resultado do volume $\left(\mathrm{m}^{3} \cdot \mathrm{ha}^{-1}\right)$ estimado usando-se a equação de volume do próprio estrato e a equação do estrato semelhante. Os valores foram próximos, com maior diferença no talhão 1, que apresentou o volume estimado pela equação semelhante igual a 3,58\% abaixo do volume estimado pela equação própria. Em termos de volume total e considerando os cinco talhões, a diferença foi de $0,88 \%$, resultado esse concebido como satisfatório.

O teste Qui-quadrado resultou em um valor calculado de 0,22 , sendo o valor tabelado a $95 \%$ de probabilidade de 11,07, ou seja, os volumes estimados são iguais, demonstrando que o método da similaridade de perfis tem aplicação prática na determinação de volume para estratos que não tenham equação própria de volume.

Diante do que foi apresentado, o procedimento proposto denominado método da similaridade de perfis pode ser resumido da seguinte forma:
1 - Abate e cubagem de árvores de clones comerciais, ou importação de bases de dados de clones comerciais.

2 - Ajuste de equações de volume de cada estrato de cubagem.

3 - Ajuste do modelo de Kozak de cada árvore cubada.

4 - Abate e cubagem de três árvores de clones novos, sendo uma árvore pequena, uma média e uma grande, em termos de dap e altura total, e posterior ajuste do modelo de Kozak, considerando-se as três árvores.

5 - Cálculo da distância euclidiana entre os parâmetros do modelo de Kozak do clone novo com os parâmetros de cada árvore dos clones comerciais e determinação da árvore mais similar através do menor valor da distância euclidiana.

6 - Determinação do estrato a que a árvore similar pertence, sendo identificada a equação de volume a ser aplicada no novo clone.

Tabela 6 - Estimativas do volume $\left(\mathrm{m}^{3} \cdot \mathrm{ha}^{-1}\right)$ dos talhões do clone sorteado utilizando a equação de volume de Schumacher e Hall (1933) própria e a equação do estrato semelhante

Table 6 - Estimates of the volume $\left(\mathrm{m}^{3} . h \mathrm{a}^{-1}\right)$ of the drafted clone stands using the Schumacher and Hall volume equation (1933) proper and the equation of the similar group

\begin{tabular}{cccc}
\hline Talhão & Área (ha) & \multicolumn{2}{c}{ Volume $\left(\mathrm{m}^{3} \cdot \mathrm{ha}^{-1}\right)$} \\
\cline { 3 - 4 } & & Equação \\
\cline { 2 - 4 } & & Própria & Semelhante \\
\hline 1 & 25,20 & 139,97 & 134,96 \\
2 & 23,45 & 100,73 & 100,57 \\
3 & 34,40 & 94,24 & 93,7 \\
4 & 49,30 & 105,73 & 105,22 \\
5 & 23,00 & 80,36 & $-0,16$ \\
\hline Total & 155,35 & 521,03 & $-0,57$ \\
\hline
\end{tabular}

R. Árvore, Viçosa-MG, v.33, n.1, p.133-141, 2009 


\section{CONCLUSÕES}

O procedimento proposto, denominado método da similaridade de perfis e usado para estimar volume individual de árvores de clones de eucalipto, pode ser usado também para estimar volume de árvores dos clones que não têm equações específicas. Isso resulta em maior precisão das estimativas de inventário, em comparação com estimativas obtidas por equações genéricas. O método da similaridade de perfis gera estimativas semelhantes em comparação com dados observados e estimados, tanto de árvores individuais quanto de todo um talhão.

O volume de árvores de clones novos, para os quais ainda não se tem equação de volume, pode ser determinado a partir de cubagem de três árvores desse volume (uma pequena, uma média e uma grande) e de um banco de dados de cubagem de outros clones comerciais.

\section{REFERÊNCIAS}

BIGING, G. S. Taper equations for second mixedconifers of Northean California. Forest

Science, v.30, n.4, p.1103-1117, 1984.

BURKHART, H. E. Cubic-foot volume of Loblolly Pine to any merchantable top limit. Southern Journal of Applied

Forestry, v.1, n.2, p.7-9, 1977.

CAMPOS, J. C. C.; TREVIZOL JUNIOR, T. L.; PAULA NETO, F. Ainda, sobre a seleção de equações de volume. Revista Árvore, v.9, n.2, p.115-126, 1985.

CAMPOS, J. C. C.; LEITE, H. G. Mensuração florestal: perguntas e respostas. 2.ed. Viçosa, MG: Universidade Federal de Viçosa, 2006. 470p.

CRUZ, C. D.; REGAZZI, A. J.; CARNEIRO, P. C. S. Modelos biométricos aplicados ao melhoramento genético. 3.ed. Viçosa, MG: Universidade Federal de Viçosa, 2004. v.1. 480p.
DEMAERSCHALK, J. P. Converting volume equations to compatible taper equations. Forest Science, v.18, n.3, p.241-245, 1972.

FERREIRA, M. Melhoramento e a silvicultura intensiva clonal. IPEF, v.45, p.22-30, 1992.

GARAY, L. Tropical forest utilization system VIII. A taper model for entire stem profile including buttressing. Seatlhe: Coll. Forest. Resour., Inst. Forest Prod. Univ. Wash., 1979. 64p. (Contrib., 36).

\section{GORGENS, E. Estimação do volume de} árvores utilizando redes neurais artificiais. 2006. 84f. Tese (Mestrado em Ciência Florestal) - Universidade Federal de Viçosa, Viçosa, MG, 2006.

GUIMARÃES, D. P.; LEITE, H. G. Influência do número de árvores na determinação de equação volumétrica para eucalyptus grandis. Scientia Forestalis, n.50, p.37-42, 1996.

GRAYBILL, F. A. Theory and application of the linear model. Massachusetts: Ouxburg Press, 1976. 704p.

HUSCH, B.; BEERS, T. W.; KERSHAW JUNIOR, J. A. Forest mensuration. 4.ed. Malabar: Krieger Publishing Company, 2003. 443p.

KOZAK, A.; MUNRO, D. D.; SMITH, J. G. H. Taper functions and their applications in forest inventory. Forest Chronicle, v.45, n.4, p.278-283, 1969.

LEITE, H.G.; ANDRADE, V.C.L. Uso do método da altura relativa em inventário florestal de um povoamento de Pinus. Revista Árvore, v.28, n.6, p.865-873, 2004.

NESBITT, J. E. Qui-Quadrado. São Paulo: Harbra, 1995. 33p.

SCHUMACHER, F. X.; HALL, F. S. Logaritmic expression of timber volume. Journal of Agricultural Research, v.47, n.9, p.719-734, 1933. 